\title{
EXACTNESS OF IDEAL TRANSFORMS AND ANNIHILATORS OF TOP LOCAL COHOMOLOGY MODULES
}

\author{
KAMAL BAHMANPOUR
}

\begin{abstract}
Let $(R, \mathfrak{m})$ be a commutative Noetherian local domain, $M$ a non-zero finitely generated $R$-module of dimension $n>0$ and $I$ be an ideal of $R$. In this paper it is shown that if $x_{1}, \ldots, x_{t}(1 \leq t \leq n)$ be a subset of a system of parameters for $M$, then the $R$-module $H_{\left(x_{1}, \ldots, x_{t}\right)}^{t}(R)$ is faithful, i.e., Ann $H_{\left(x_{1}, \ldots, x_{t}\right)}^{t}(R)=0$. Also, it is shown that, if $H_{I}^{i}(R)=0$ for all $i>\operatorname{dim} R-\operatorname{dim} R / I$, then the $R$-module $H_{I}^{\operatorname{dim} R-\operatorname{dim} R / I}(R)$ is faithful. These results provide some partially affirmative answers to the Lynch's conjecture in [10]. Moreover, for an ideal $I$ of an arbitrary Noetherian ring $R$, we calculate the annihilator of the top local cohomology module $H_{I}^{1}(M)$, when $H_{I}^{i}(M)=0$ for all integers $i>1$. Also, for such ideals we show that the finitely generated $R$-algebra $D_{I}(R)$ is a flat $R$ algebra.
\end{abstract}

\section{Introduction}

Throughout this paper, let $R$ denote a commutative Noetherian ring (with identity) and $I$ an ideal of $R$. The local cohomology modules $H_{I}^{i}(M), i=$ $0,1,2, \ldots$, of an $R$-module $M$ with respect to $I$ were introduced by Grothendieck, [7]. They arise as the derived functors of the left exact functor $\Gamma_{I}(-)$, where for an $R$-module $M, \Gamma_{I}(M)$ is the submodule of $M$ consisting of all elements annihilated by some power of $I$, i.e., $\bigcup_{n=1}^{\infty}\left(0:_{M} I^{n}\right)$. There is a natural isomorphism:

$$
H_{I}^{i}(M) \cong \lim _{n \geq 1} \operatorname{Ext}_{R}^{i}\left(R / I^{n}, M\right) .
$$

We refer the reader to [7] or [4] for more details about local cohomology.

One of the important and hard problems in commutative algebra is determining the annihilator of the local cohomology module $H_{I}^{i}(M)$. This problem

Received January 2, 2015; Revised April 22, 2015.

2010 Mathematics Subject Classification. 13D45, 14B15, $13 \mathrm{E} 05$.

Key words and phrases. cohomological dimension, ideal transform, local cohomology, Noetherian ring.

This research of the author was supported by a grant from IPM (No. 93130022). 
has been studied by several authors; see for example [1], [2], [9], [10], [11], [15], [16] and [18].

Recall that, for an $R$-module $M$, the cohomological dimension of $M$ with respect to $I$ is defined as

$$
\operatorname{cd}(I, M):=\max \left\{i \in \mathbb{Z}: H_{I}^{i}(M) \neq 0\right\} .
$$

In [10] Lynch conjectured the following:

Conjecture. For every Noetherian local ring $(R, \mathfrak{m})$ and any ideal $I$ of $R$, if $\operatorname{cd}(I, R)=t>0$, then

$$
\operatorname{dim} R / \operatorname{Ann} H_{I}^{t}(R)=\operatorname{dim} R / \Gamma_{I}(R)
$$

Lynch has shown that this conjecture has affirmative answer in several cases. In particular, in each of the following cases it is shown that this conjecture holds:

(i) $\operatorname{cd}(I, R)=\operatorname{dim} R>0$,

(ii) $\operatorname{cd}(I, R)=\operatorname{grade}(I, R)>0$.

One other interesting situation where this conjecture holds is the case where $R$ is a regular local ring containing a field. In the introduction of [8] this is mentioned that for such rings the injective dimension of top local cohomology $H_{I}^{t}(R)$ does not exceed the $\operatorname{dim} \operatorname{Supp} H_{I}^{t}(R)$. This result implies that for each minimal element $\mathfrak{p}$ of $\operatorname{Supp} H_{I}^{t}(R)$, the $R_{\mathfrak{p}}$-module $H_{I R_{\mathfrak{p}}}^{t}\left(R_{\mathfrak{p}}\right)$ is injective and so $H_{I}^{t}(R)$ is a faithful $R$-module, i.e., Ann $H_{I}^{t}(R)=0$.

If this conjecture has an affirmative answer in general, then for any non-zero and proper ideal $I$ of any Noetherian local domain, the top local cohomology module $H_{I}^{t}(R)$ is faithful.

Pursuing this point of view further we establish some results about the annihilator of top local cohomology modules. The main new case that we consider in this paper, is the case where the ideal $I$ is generated by a subset of a system of parameters for some finitely generated $R$-modules. For such ideals of any Noetherian local domain we present an affirmative answer to the Lynch's conjecture. Also, we shall prove the conjecture for the special case that $R$ is Noetherian local domain and $\operatorname{cd}(I, R)=\operatorname{dim} R-\operatorname{dim} R / I$. This result can be useful for the non-catenary Noetherian local domains, specially for the ideals $I$ with height $(I)<\operatorname{dim} R-\operatorname{dim} R / I$. Finally, in this direction we compute the annihilator of $H_{I}^{1}(R)$, in the case where $I$ is an ideal of an arbitrary Noetherian ring $R$, with $\operatorname{cd}(I, R)=1$. All of these results will be proved in Section 2 .

In Section 3 of this paper we will study the exactness of the $I$-transform functor $D_{I}(-)=\lim _{n \geq 1} \operatorname{Hom}_{R}\left(I^{n},-\right)$. It is well known that, when the $I$ transform functor $\overrightarrow{D_{I}}(-)$ is exact, then the $R$-module $D_{I}(R)$ has a finitely generated $R$-algebra structure. In Section 3 , as our main result, we shall prove that, if the $I$-transform functor $D_{I}(-)$ is exact then the finitely generated $R$-algebra $D_{I}(R)$ is also a flat $R$-algebra. Moreover, we shall present some conditions for the exactness of the ideal transforms. 
Recall that, the arithmetic rank of the ideal $I$, denoted by $\operatorname{ara}(I)$, is the least number of elements of $I$ required to generate an ideal which has the same radical as $I$, i.e.,

$$
\operatorname{ara}(I):=\min \left\{n \in \mathbb{N}_{0}: \exists x_{1}, \ldots, x_{n} \in I \text { with } \operatorname{Rad}\left(\left(x_{1}, \ldots, x_{n}\right)\right)=\operatorname{Rad}(I)\right\} .
$$

For each $R$-module $L$, we denote by $\operatorname{Assh}_{R} L$ the set $\left\{\mathfrak{p} \in \operatorname{Ass}_{R} L: \operatorname{dim} R / \mathfrak{p}=\right.$ $\operatorname{dim} L\}$. For any prime ideal $\mathfrak{p}$ of $R$ and any positive integer $k$ we denote the $k^{\text {th }}$ symbolic power of $\mathfrak{p}$ by $\mathfrak{p}^{(k)}$. For every non-zero $R$-module $T$, we denote the set of all zero-divisors of $T$ in $R$ by $Z_{R}(T)$. Also, for any ideal $\mathfrak{a}$ of $R$, we denote $\{\mathfrak{p} \in \operatorname{Spec} R: \mathfrak{p} \supseteq \mathfrak{a}\}$ by $V(\mathfrak{a})$. Finally, for any ideal $\mathfrak{b}$ of $R$, the radical of $\mathfrak{b}$, denoted by $\operatorname{Rad}(\mathfrak{b})$, is defined to be the set $\left\{x \in R: x^{n} \in \mathfrak{b}\right.$ for some $n \in \mathbb{N}\}$. For any unexplained notation and terminology we refer the reader to $[12]$ or $[4]$.

\section{Annihilators of top local cohomology modules}

In this section we present some partially affirmative answers to the Lynch's conjecture in [10]. The main goals of this section are Theorems 2.2, 2.3, 2.7 and 2.9 .

For an $R$-module $M$ of finite dimension we define the submodule $T_{R}(M)$ of $M$ as follows:

$$
T_{R}(M):=\cup\{N: N \leq M \text { and } \operatorname{dim} N<\operatorname{dim} M\} .
$$

It is clear that $\operatorname{Ass}_{R} M / T_{R}(M)=\operatorname{Assh}_{R} M$. The following result which is needed in the proof of the main result of this section has been proved in [2].

Lemma 2.1. Let $(R, \mathfrak{m})$ be a Noetherian local ring of dimension $d \geq 1$. Then

$$
\operatorname{Ann} H_{\mathfrak{m}}^{d}(R)=T_{R}(R)
$$

Proof. See [2, Theorem 2.8].

The following result is the first main result of this paper. Note that the part (v) of this result is an special case of Lynch's conjecture.

Theorem 2.2. Let $(R, \mathfrak{m})$ be a Noetherian local ring and $M$ be a non-zero finitely generated $R$-module of dimension $n \geq 1$. Let $x_{1}, \ldots, x_{t} \in \mathfrak{m},(1 \leq t \leq$ $n)$ be a part of a system of parameters for $M$. Then the following statements hold:

(i) $\operatorname{cd}\left(\left(x_{1}, \ldots, x_{t}\right), M\right)=t$,

(ii) $\operatorname{dim} H_{\left(x_{1}, \ldots, x_{t}\right)}^{t}(M)=n-t$,

(iii) $\operatorname{Ann} H_{\left(x_{1}, \ldots, x_{t}\right)}^{t}(M) \subseteq \bigcap_{\mathfrak{p} \in \operatorname{Assh}_{R} M} \mathfrak{p}$,

(iv) Ann $H_{\left(x_{1}, \ldots, x_{t}\right)}^{t}(R) \subseteq \bigcap_{\mathfrak{p} \in \operatorname{Assh}_{R} M} \bigcap_{k=1}^{\infty} \mathfrak{p}^{(k)} \subseteq Z_{R}(R)$,

(v) If $R$ is a domain, then we have Ann $H_{\left(x_{1}, \ldots, x_{t}\right)}^{t}(R)=0$. 
Proof. (i) and (ii). Since Supp $M=\operatorname{Supp} R / \operatorname{Ann} M$ it follows from [5, Theorem 2.2] that $\operatorname{cd}\left(\left(x_{1}, \ldots, x_{t}\right), M\right)=\operatorname{cd}\left(\left(x_{1}, \ldots, x_{t}\right), R /\right.$ Ann $\left.M\right)$. Moreover, as

$$
\overline{x_{1}}=x_{1}+\operatorname{Ann} M, \ldots, \overline{x_{t}}=x_{t}+\operatorname{Ann} M \in R / \operatorname{Ann} M
$$

is a part of a system of parameters for the local $\operatorname{ring} R / \operatorname{Ann} M$, it follows from [3, Proposition 3.2] and [4, Theorem 4.2.1], that

$$
\begin{aligned}
\operatorname{cd}\left(\left(x_{1}, \ldots, x_{t}\right), M\right) & =\operatorname{cd}\left(\left(x_{1}, \ldots, x_{t}\right), R / \operatorname{Ann} M\right) \\
& =\operatorname{cd}\left(\left(\overline{x_{1}}, \ldots, \overline{x_{t}}\right), R / \operatorname{Ann} M\right)=t .
\end{aligned}
$$

Also, as Supp $M=\operatorname{Supp} R / \operatorname{Ann} M$, using localization, it follows from [5, Theorem 2.2] and [4, Theorem 4.3.2], that

$$
\text { Supp } H_{\left(x_{1}, \ldots, x_{t}\right)}^{t}(M)=\operatorname{Supp} H_{\left(x_{1}, \ldots, x_{t}\right)}^{t}(R / \operatorname{Ann} M) .
$$

Now, (ii) follows from [3, Proposition 3.2], using [4, Theorem 4.2.1].

We can find elements $x_{t+1}, \ldots, x_{n} \in \mathfrak{m}$ such that $x_{1}, \ldots, x_{n}$ is a system of parameters for $M$. Then using [19, Corollary 3.5] and [4, Theorem 3.3.1], we have the following:

$$
\begin{aligned}
H_{R x_{t+1}}^{1}\left(H_{\left(x_{1}, \ldots, x_{t}\right)}^{t}(M)\right) \cong & H_{\left(x_{1}, \ldots, x_{t+1}\right)}^{t+1}(M), \\
H_{R x_{t+2}}^{1}\left(H_{\left(x_{1}, \ldots, x_{t+1}\right)}^{t+1}(M)\right) \cong & H_{\left(x_{1}, \ldots, x_{t+2}\right)}^{t+2}(M) \\
\cong & H_{\left(x_{t+1}, x_{t+2}\right)}^{2}\left(H_{\left(x_{1}, \ldots, x_{t}\right)}^{t}(M)\right), \\
& \vdots \\
H_{R x_{n}}^{1}\left(H_{\left(x_{1}, \ldots, x_{n-1}\right)}^{n-1}(M)\right) \cong & H_{\left(x_{1}, \ldots, x_{n}\right)}^{n}(M) \\
\cong & H_{\left(x_{t+1}, x_{t+2}, \ldots, x_{n}\right)}^{n-t}\left(H_{\left(x_{1}, \ldots, x_{t}\right)}^{t}(M)\right) .
\end{aligned}
$$

But by [4, Theorem 4.2.1] we have

$$
H_{\left(x_{1}, \ldots, x_{n}\right)}^{n}(M) \cong H_{\left(x_{1}, \ldots, x_{n}\right)+\operatorname{Ann} M / \operatorname{Ann} M}^{n}(M) \cong H_{\mathfrak{m} / \operatorname{Ann} M}^{n}(M) \cong H_{\mathfrak{m}}^{n}(M) .
$$

Now, for any $\mathfrak{p} \in \operatorname{Assh}_{R} M$ by [4, Theorem 7.3.2], we have $\mathfrak{p} \in \operatorname{Att} H_{\mathfrak{m}}^{n}(M)$, which implies that Ann $H_{\mathfrak{m}}^{n}(M) \subseteq \mathfrak{p}$. Thus we have

$$
\begin{aligned}
\operatorname{Ann} H_{\left(x_{1}, \ldots, x_{t}\right)}^{t}(M) & \subseteq \operatorname{Ann} H_{\left(x_{t+1}, x_{t+2}, \ldots, x_{n}\right)}^{n-t}\left(H_{\left(x_{1}, \ldots, x_{t}\right)}^{t}(M)\right) \\
& =\operatorname{Ann} H_{\mathfrak{m}}^{n}(M) \subseteq \mathfrak{p} .
\end{aligned}
$$

This completes the proof of (iii).

(iv) Let $\mathfrak{p} \in \operatorname{Assh}_{R} M$ and $x_{1}, \ldots, x_{n}$ be as the same in the proof of (iii). Then for every positive integer $k$, it is clear that $x_{1}, \ldots, x_{n}$ is a system of parameters for the $R$-module $R / \mathfrak{p}^{(k)}$. Then applying the method used in the proof of (iii) it follows that

$$
H_{\left(x_{t+1}, x_{t+2}, \ldots, x_{n}\right)}^{n-t}\left(H_{\left(x_{1}, \ldots, x_{t}\right)}^{t}\left(R / \mathfrak{p}^{(k)}\right)\right) \cong H_{\mathfrak{m}}^{n}\left(R / \mathfrak{p}^{(k)}\right) .
$$

So, using Lemma 2.1 we have

$$
\operatorname{Ann} H_{\left(x_{1}, \ldots, x_{t}\right)}^{t}\left(R / \mathfrak{p}^{(k)}\right) \subseteq \operatorname{Ann} H_{\left(x_{t+1}, x_{t+2}, \ldots, x_{n}\right)}^{n-t}\left(H_{\left(x_{1}, \ldots, x_{t}\right)}^{t}\left(R / \mathfrak{p}^{(k)}\right)\right)
$$




$$
\begin{aligned}
& =\operatorname{Ann} H_{\mathfrak{m}}^{n}\left(R / \mathfrak{p}^{(k)}\right) \\
& =\operatorname{Ann} H_{\mathfrak{m} / \mathfrak{p}^{(k)}}^{n}\left(R / \mathfrak{p}^{(k)}\right) \\
& =\mathfrak{p}^{(k)} .
\end{aligned}
$$

On the other hand, the exact sequence

$$
0 \rightarrow \mathfrak{p}^{(k)} \rightarrow R \rightarrow R / \mathfrak{p}^{(k)} \rightarrow 0
$$

induces the following exact sequence

$$
H_{\left(x_{1}, \ldots, x_{t}\right)}^{t}(R) \rightarrow H_{\left(x_{1}, \ldots, x_{t}\right)}^{t}\left(R / \mathfrak{p}^{(k)}\right) \rightarrow H_{\left(x_{1}, \ldots, x_{t}\right)}^{t+1}\left(\mathfrak{p}^{(k)}\right) .
$$

But, in view of [4, Theorem 3.3.1], we have

$$
H_{\left(x_{1}, \ldots, x_{t}\right)}^{t+1}\left(\mathfrak{p}^{(k)}\right)=0
$$

Hence we have the following exact sequence

$$
H_{\left(x_{1}, \ldots, x_{t}\right)}^{t}(R) \rightarrow H_{\left(x_{1}, \ldots, x_{t}\right)}^{t}\left(R / \mathfrak{p}^{(k)}\right) \rightarrow 0
$$

which implies that

$$
\operatorname{Ann} H_{\left(x_{1}, \ldots, x_{t}\right)}^{t}(R) \subseteq \operatorname{Ann} H_{\left(x_{1}, \ldots, x_{t}\right)}^{t}\left(R / \mathfrak{p}^{(k)}\right) \subseteq \mathfrak{p}^{(k)} .
$$

So we have

$$
\operatorname{Ann} H_{\left(x_{1}, \ldots, x_{t}\right)}^{t}(R) \subseteq \bigcap_{\mathfrak{p} \in \operatorname{Assh}_{R}} \bigcap_{M=1}^{\infty} \mathfrak{p}^{(k)} .
$$

In order to prove the inclusion,

$$
\bigcap_{\mathfrak{p} \in \operatorname{Assh}_{R}} \bigcap_{k=1}^{\infty} \mathfrak{p}^{(k)} \subseteq Z_{R}(R),
$$

it is enough to show that $\bigcap_{k=1}^{\infty} \mathfrak{p}^{(k)} \subseteq Z_{R}(R)$, for every prime ideal $\mathfrak{p}$ of $R$. To do this, let $\varphi: R \rightarrow R_{\mathfrak{p}}$ be the natural homomorphism. Then, since for each positive integer $k$ by the definition we have $\mathfrak{p}^{(k)}=\varphi^{-1}\left(\mathfrak{p}^{k} R_{\mathfrak{p}}\right)$ and by Krull's Intersection Theorem, we have $\bigcap_{k=1}^{\infty} \mathfrak{p}^{k} R_{\mathfrak{p}}=0$ it follows that $\varphi\left(\bigcap_{k=1}^{\infty} \mathfrak{p}^{(k)}\right)=$ 0 . So, as the ideal $J:=\bigcap_{k=1}^{\infty} \mathfrak{p}^{(k)}$ is finitely generated, it is straightforward and so left to reader, that $s J=0$ for some element $s \in(R \backslash \mathfrak{p})$ and hence $\bigcap_{k=1}^{\infty} \mathfrak{p}^{(k)} \subseteq Z_{R}(R)$

(v) Since $R$ is a domain it follows from the definition that $Z_{R}(R)=0$ and so the assertion follows from part (iv).

The following result is a consequence of Theorem 2.2 .

Theorem 2.3. Let $(R, \mathfrak{m})$ be a Noetherian local ring of dimension $d \geq 1$ and $x_{1}, \ldots, x_{t} \in \mathfrak{m},(1 \leq t \leq d)$ be a part of a system of parameters for $R$. Then the following statements hold:

(i) $\operatorname{cd}\left(\left(x_{1}, \ldots, x_{t}\right), R\right)=t$,

(ii) $\operatorname{dim} R / \operatorname{Ann} H_{\left(x_{1}, \ldots, x_{t}\right)}^{t}(R)=\operatorname{dim} R=\operatorname{dim} R / \Gamma_{\left(x_{1}, \ldots, x_{t}\right)}(R)$. 
Proof. The statement (i) follows from Theorem 2.2. In order to prove (ii), let $\mathfrak{p} \in \operatorname{Assh}_{R} R$. Then, by Theorem 2.2 we have

$$
\operatorname{Ann} H_{\left(x_{1}, \ldots, x_{t}\right)}^{t}(R) \subseteq \bigcap_{k=1}^{\infty} \mathfrak{p}^{(k)} \subseteq \mathfrak{p} .
$$

So, we have

$$
\operatorname{dim} R=\operatorname{dim} R / \mathfrak{p} \leq \operatorname{dim} R / \operatorname{Ann} H_{\left(x_{1}, \ldots, x_{t}\right)}^{t}(R) \leq \operatorname{dim} R .
$$

Therefore, $\operatorname{dim} R / \operatorname{Ann} H_{\left(x_{1}, \ldots, x_{t}\right)}^{t}(R)=\operatorname{dim} R$. On the other hand, by definition there exists a positive integer $k$ such that $\left(x_{1}, \ldots, x_{t}\right)^{k} \Gamma_{\left(x_{1}, \ldots, x_{t}\right)}(R)=0 \subseteq \mathfrak{p}$. Now, since $\left(x_{1}, \ldots, x_{t}\right) \nsubseteq \mathfrak{p}$ it follows that $\Gamma_{\left(x_{1}, \ldots, x_{t}\right)}(R) \subseteq \mathfrak{p}$. Therefore, we have

$$
\operatorname{dim} R=\operatorname{dim} R / \mathfrak{p} \leq \operatorname{dim} R / \Gamma_{\left(x_{1}, \ldots, x_{t}\right)}(R) \leq \operatorname{dim} R,
$$

which implies that

$$
\operatorname{dim} R / \Gamma_{\left(x_{1}, \ldots, x_{t}\right)}(R)=\operatorname{dim} R=\operatorname{dim} R / \operatorname{Ann} H_{\left(x_{1}, \ldots, x_{t}\right)}^{t}(R) .
$$

Recall that for each $R$-module $M$, all integers $j \geq 0$ and all prime ideals $\mathfrak{p}$ of $R$, the $j^{\text {th }}$ Bass number of $M$ with respect to $\mathfrak{p}$ is defined as $\mu_{R}^{j}(\mathfrak{p}, M)=$ $\operatorname{dim}_{k(\mathfrak{p})} \operatorname{Ext}_{R_{\mathfrak{p}}}^{j}\left(k(\mathfrak{p}), M_{\mathfrak{p}}\right)$, where $k(\mathfrak{p}):=R_{\mathfrak{p}} / \mathfrak{p} R_{\mathfrak{p}}$.

Proposition 2.4. Let $(R, \mathfrak{m})$ be a Noetherian local domain and $I$ be a proper non-zero ideal of $R$. Let $t$ be a positive integer such that the $R$-module $H_{I}^{t}(R)$ is not faithful, i.e., Ann $H_{I}^{t}(R) \neq 0$. Then for every finitely generated $R$-module $M$ the $R$-module $H_{I}^{t}(M)$ is not faithful.

Proof. If $M$ is not faithful, then as Ann $M \subseteq$ Ann $H_{I}^{t}(M)$ there is nothing to prove. So we may assume that $M$ is faithful. Then we have $\mu^{0}(0, M) \neq 0$. Let $n:=\mu^{0}(0, M)$. Then by $[17$, Lemma 2.1$]$ there exists an exact sequence

$$
0 \rightarrow \oplus_{i=1}^{n} R \rightarrow M \rightarrow T \rightarrow 0
$$

for some finitely generated $R$-module $T$. Let $S:=R \backslash\{0\}$. Then according to the definition $S^{-1} M$ is a $n$-dimensional vector space over the field $K=S^{-1} R$. Therefore, it follows from the exact sequence

$$
0 \rightarrow \oplus_{i=1}^{n} S^{-1} R \rightarrow S^{-1} M \rightarrow S^{-1} T \rightarrow 0
$$

that $S^{-1} T=0$ and so Ann $T \neq 0$. Let $0 \neq a \in \operatorname{Ann} H_{I}^{t}(R)$ and $0 \neq b \in \operatorname{Ann} T$. The exact sequence (2.4.1) yields the exact sequence

$$
H_{I}^{t}\left(\oplus_{i=1}^{n} R\right) \rightarrow H_{I}^{t}(M) \rightarrow H_{I}^{t}(T) .
$$

Now since $H_{I}^{t}\left(\oplus_{i=1}^{n} R\right) \cong \oplus_{i=1}^{n} H_{I}^{t}(R)$, the exact sequence (2.4.2) implies that $0 \neq a b \in \operatorname{Ann} H_{I}^{t}(M)$ and so $H_{I}^{t}(M)$ is not faithful.

The following lemma is needed in the proof of Proposition 2.6. 
Lemma 2.5. Let $(R, \mathfrak{m})$ be a Noetherian local domain and $I$ be a proper nonzero ideal of $R$. Let $t$ be a positive integer and $\Sigma_{0}$ be a non-zero $R$-module such that the $R$-module $H_{I}^{t}\left(\Sigma_{0}\right)$ is faithful. Then the $R$-module

$$
D=\bigoplus_{i=t}^{\operatorname{cd}(I, R)} H_{I}^{i}(R)
$$

is faithful.

Proof. Suppose that the contrary is true. Then there is an element $0 \neq a \in \mathfrak{m}$ such that $a D=0$. Let $A$ be a subset of $\Sigma_{0}$ which generates the $R$-module $\Sigma_{0}$. Then, $\Sigma_{0}$ is a homomorphic image of the free $R$-module $F_{0}=\bigoplus_{\gamma \in A} R$. So there is an exact sequence

$$
0 \rightarrow \Sigma_{1} \rightarrow F_{0} \stackrel{f_{0}}{\rightarrow} \Sigma_{0} \rightarrow 0
$$

which induces the exact sequence

$$
H_{I}^{t}\left(F_{0}\right) \rightarrow H_{I}^{t}\left(\Sigma_{0}\right) \rightarrow H_{I}^{t+1}\left(\Sigma_{1}\right)
$$

Since $H_{I}^{t}\left(F_{0}\right) \cong \bigoplus_{\gamma \in A} H_{I}^{t}(R)$ it follows that $a H_{I}^{t}\left(F_{0}\right)=0$. Let $b \in \operatorname{Ann} H_{I}^{t+1}\left(\Sigma_{1}\right)$. Then, by the exact sequence (2.5.1) we have $a b \in \operatorname{Ann} H_{I}^{t}\left(\Sigma_{0}\right)=0$ and so $b=0$. Therefore, the $R$-module $H_{I}^{t+1}\left(\Sigma_{1}\right)$ is faithful. In particular, $\mathrm{H}_{\mathrm{I}}^{\mathrm{t}+1}\left(\Sigma_{1}\right) \neq 0$ and $\Sigma_{1}$ is not a free $R$-module, (Because $a \notin \operatorname{Ann} H_{I}^{t+1}\left(\Sigma_{1}\right)=0$ ). Now, let

$$
\mathbf{F}^{\bullet}: \quad \cdots \rightarrow F_{2} \stackrel{f_{2}}{\rightarrow} F_{1} \stackrel{f_{1}}{\rightarrow} F_{0} \stackrel{f_{0}}{\rightarrow} \Sigma_{0} \rightarrow 0
$$

be a free resolution for the $R$-module $\Sigma_{0}$. Considering the short exact sequences

$$
0 \rightarrow \Sigma_{n+1} \rightarrow F_{n} \rightarrow \Sigma_{n} \rightarrow 0,
$$

where $\Sigma_{j}=\operatorname{im}\left(f_{j}\right)$ for $j=0,1,2, \ldots$, and applying the same method, we can argue by induction on $n$ that the $R$-module $H_{I}^{t+n}\left(\Sigma_{n}\right)$ is faithful. In particular, $H_{I}^{t+n}\left(\Sigma_{n}\right) \neq 0$ and $\Sigma_{n}$ is not a free $R$-module. But, this is a contradiction, because according to the Grothendieck's Vanishing Theorem we have $H_{I}^{i}\left(\Sigma_{i-t}\right)=0$ for each $i \geq 1+\operatorname{dim} R$. So, the $R$-module $D$ is faithful.

The following proposition plays a key role in the proof of Theorem 2.7.

Proposition 2.6. Let $(R, \mathfrak{m})$ be a Noetherian local domain and $I$ be a proper non-zero ideal of $R$. Then the $R$-module

$$
D=\bigoplus_{i=\operatorname{dim} R-\operatorname{dim} R / I}^{\operatorname{cd}(I, R)} H_{I}^{i}(R)
$$

is faithful.

Proof. Let $\operatorname{dim} R=d$ and $t=d-\operatorname{dim} R / I$. Then $I$ contains a subset $x_{1}, \ldots, x_{t}$ of a system of parameters for $R$. Hence there exist elements $y_{1}, \ldots, y_{d-t}$ in $R$ such that $x_{1}, \ldots, x_{t}, y_{1}, \ldots, y_{d-t}$ is a system of parameters for $R$. Now, since $\operatorname{dim} R /\left(y_{1}, \ldots, y_{d-t}\right)=t$ it follows that for every $\mathfrak{p} \in \operatorname{Assh}_{R} R /\left(y_{1}, \ldots, y_{d-t}\right)$ 
and any positive integer $k$ we have $\operatorname{dim} R / \mathfrak{p}^{(k)}=t$. Thus, using [4, Exercise 2.1.9] we have

$$
H_{I}^{t}\left(R / \mathfrak{p}^{(k)}\right) \cong H_{I+\mathfrak{p}^{(k)}}^{t}\left(R / \mathfrak{p}^{(k)}\right)=H_{\mathfrak{m}}^{t}\left(R / \mathfrak{p}^{(k)}\right) .
$$

Therefore, by Lemma 2.1 we have Ann $H_{I}^{t}\left(R / \mathfrak{p}^{(k)}\right)=\mathfrak{p}^{(k)}$. Next, let $\Sigma_{0}:=$ $\bigoplus_{k=1}^{\infty} R / \mathfrak{p}^{(k)}$. Then, as $H_{I}^{t}\left(\Sigma_{0}\right) \cong \bigoplus_{k=1}^{\infty} H_{I}^{t}\left(R / \mathfrak{p}^{(k)}\right)$ it follows that

$$
\text { Ann } H_{I}^{t}\left(\Sigma_{0}\right)=\bigcap_{k=1}^{\infty} \mathfrak{p}^{(k)} .
$$

But, since $R$ is a domain it follows that $\bigcap_{k=1}^{\infty} \mathfrak{p}^{(k)}=0$ and so $H_{I}^{t}\left(\Sigma_{0}\right)$ is a faithful $R$-module. Now the assertion is clear by Lemma 2.5 .

The following result provides another partially affirmative answer to the Lynch's conjecture.

Theorem 2.7. Let $(R, \mathfrak{m})$ be a Noetherian local domain and $I$ be a proper nonzero ideal of $R$. If $\operatorname{cd}(I, R)=\operatorname{dim} R-\operatorname{dim} R / I$, then the $R$-module $H_{I}^{\operatorname{cd}(I, R)}(R)$ is faithful.

Proof. Follows from Proposition 2.6.

The following lemma is needed in the proof of Theorem 2.9.

Lemma 2.8. Let $(R, \mathfrak{m})$ be a Noetherian local ring, $I$ be an ideal of $R$ and $M$ be a finitely generated $R$-module. If $\operatorname{cd}(I, M)=t \geq 1$, then $H_{I}^{t}(M)=I H_{I}^{t}(M)$. In particular, the $R$-module $H_{I}^{t}(M)$ is not finitely generated.

Proof. Since $M$ has a finitely generated $R / A n n M$-module structure, it follows that there is an exact sequence

$$
0 \rightarrow K \rightarrow \bigoplus_{i=1}^{k} R / \operatorname{Ann} M \stackrel{\pi}{\rightarrow} M \rightarrow 0,
$$

for some positive integer $k$, where $K$ is the kernel of the epimorphism $\pi$. But the exact sequence (2.8.1) yields the exact sequence

$$
H_{I}^{t}\left(\bigoplus_{i=1}^{k} R / \operatorname{Ann} M\right) \rightarrow H_{I}^{t}(M) \rightarrow H_{I}^{t+1}(K) .
$$

Since, Supp $K \subseteq \operatorname{Supp} R / \operatorname{Ann} M=\operatorname{Supp} M$, it follows from [5, Theorem 2.2] that $\operatorname{cd}(I, K) \leq \operatorname{cd}(I, M)=t$ and hence $H_{I}^{t+1}(K)=0$. Therefore, from the exact sequence (2.8.2) we get the exact sequence

$$
\bigoplus_{i=1}^{k} H_{I}^{t}(R / \operatorname{Ann} M) \rightarrow H_{I}^{t}(M) \rightarrow 0
$$

which implies that, in order to prove $H_{I}^{t}(M)=I H_{I}^{t}(M)$, it is enough to prove

$$
H_{I}^{t}(R / \operatorname{Ann} M)=I H_{I}^{t}(R / \operatorname{Ann} M) .
$$


To do this, as $\operatorname{Supp} R / \operatorname{Ann} M=\operatorname{Supp}(M)$, it follows from [5, Theorem 2.2] that, $\operatorname{cd}(I, R / \operatorname{Ann} M)=\operatorname{cd}(I, M)=t$. Moreover, in view of $[4$, Theorem 4.2.1], we have $\operatorname{cd}(I, R / \operatorname{Ann} M)=\operatorname{cd}((I+\operatorname{Ann} M) / \operatorname{Ann} M, R / \operatorname{Ann} M)$. So, without loss of generality, replacing the $\operatorname{ring} R$ with the $\operatorname{ring} R / \operatorname{Ann} M$, we may assume that $M=R$ and $\operatorname{cd}(I, R)=t \geq 1$. Now by [4, Exercise 6.1 .8 and Corollary 2.1.7], we have

$$
H_{I}^{t}(R) / I H_{I}^{t}(R) \cong H_{I}^{t}(R) \otimes_{R} R / I \cong H_{I}^{t}(R / I)=0 .
$$

So, $H_{I}^{t}(R)=I H_{I}^{t}(R)$. Now, the second assertion follows from Nakayama's Lemma.

The following theorem is the final main result of this section.

Theorem 2.9. Let $R$ be a Noetherian ring, $I$ an ideal of $R$ and $M$ be a finitely generated $R$-module. Let $A=\operatorname{Ass}_{R} M \cap V(I)$ and $B=\left\{\mathfrak{p} \in \operatorname{Ass}_{R} M\right.$ : $\mathfrak{p}+I=R\}$. If $\operatorname{cd}(I, M)=1$, then $\operatorname{Ann} H_{I}^{1}(M)=\operatorname{Ann} M / \Gamma_{J}(M)$, where $J=$ $\bigcap_{\mathfrak{p} \in(A \cup B)} \mathfrak{p}$.

Proof. First we show that $H_{I}^{i}\left(\Gamma_{J}(M)\right)=0$ for all integers $i \geq 1$. Assume the contrary. Then there is a positive integer $j \geq 1$ such that $H_{I}^{j}\left(\Gamma_{J}(M)\right) \neq 0$. Then, in view of [12, Theorem 6.4], there exists a chain $0=L_{0} \subset L_{1} \subset \cdots \subset$ $L_{k-1} \subset L_{k}=\Gamma_{J}(M)$ of submodules of $\Gamma_{J}(M)$ such that for each $1 \leq i \leq k$ we have $L_{i} / L_{i-1} \simeq R / \mathfrak{Q}_{i}$, with $\mathfrak{Q}_{i} \in \operatorname{Spec} R$. Now, using the exact sequences

$$
0 \rightarrow L_{i-1} \rightarrow L_{i} \rightarrow R / \mathfrak{Q}_{i} \rightarrow 0
$$

for $i=1, \ldots, k$, we get the exact sequences

$$
H_{I}^{j}\left(L_{i-1}\right) \rightarrow H_{I}^{j}\left(L_{i}\right) \rightarrow H_{I}^{j}\left(R / \mathfrak{Q}_{i}\right)
$$

for $i=1, \ldots, k$. Now, using the fact that $H_{I}^{j}\left(L_{k}\right)=H_{I}^{j}\left(\Gamma_{J}(M)\right) \neq 0$, we can deduce that $H_{I}^{j}(R / \mathfrak{q}) \neq 0$ for some $\mathfrak{q} \in\left\{\mathfrak{Q}_{1}, \ldots, \mathfrak{Q}_{k}\right\}$. But, it is easy to see that $\left\{\mathfrak{Q}_{1}, \ldots, \mathfrak{Q}_{k}\right\} \subseteq \operatorname{Supp} \Gamma_{J}(M)$. So, we have $H_{I}^{j}(R / \mathfrak{q}) \neq 0$ for some $\mathfrak{q} \in \operatorname{Supp} M$. Then, we have $\mathfrak{q} \in V(\mathfrak{p})$ for some $\mathfrak{p} \in \operatorname{Ass}_{R} \Gamma_{J}(M)$. Since $J \subseteq \operatorname{Rad}\left(\operatorname{Ann} \Gamma_{J}(M)\right) \subseteq \mathfrak{p}$, it follows that there exists an element $\mathfrak{p}_{1} \in(A \cup B)$ such that $\mathfrak{p}_{1} \subseteq \mathfrak{p}$ and so $\mathfrak{p}_{1} \subseteq \mathfrak{q}$. If $\mathfrak{p}_{1} \in A$, then $I \subseteq \mathfrak{p}_{1} \subseteq \mathfrak{q}$, which means $R / \mathfrak{q}$ is $I$-torsion and hence by [4, Corollary 2.1.7], we have $H_{I}^{j}(R / \mathfrak{q})=0$, which is a contradiction. So we have $\mathfrak{p}_{1} \in B$. Then we have $R=\mathfrak{p}_{1}+I \subseteq \mathfrak{q}+I \subseteq R$, and so $\mathfrak{q}+I=R$. So, we have

$$
H_{I}^{j}(R / \mathfrak{q}) \cong H_{(I+\mathfrak{q}) / \mathfrak{q}}^{j}(R / \mathfrak{q})=H_{R / \mathfrak{q}}^{j}(R / \mathfrak{q}) \cong H_{R}^{j}(R / \mathfrak{q})=0,
$$

which is contradiction. (Note that $\Gamma_{R}(-)$ is the zero functor, and so $H_{R}^{i}(-)$ is the zero functor for all integers $i \geq 0$.) Hence, $H_{I}^{i}\left(\Gamma_{J}(M)\right)=0$ for all integers $i \geq 1$. Now the exact sequence

$$
0 \rightarrow \Gamma_{J}(M) \rightarrow M \rightarrow M / \Gamma_{J}(M) \rightarrow 0,
$$


yields the exact sequence

$$
H_{I}^{1}\left(\Gamma_{J}(M)\right) \rightarrow H_{I}^{1}(M) \rightarrow H_{I}^{1}\left(M / \Gamma_{J}(M)\right) \rightarrow H_{I}^{2}\left(\Gamma_{J}(M)\right),
$$

which considering the fact that $H_{I}^{1}\left(\Gamma_{J}(M)\right)=0=H_{I}^{2}\left(\Gamma_{J}(M)\right)$, provide the isomorphism $H_{I}^{1}(M) \cong H_{I}^{1}\left(M / \Gamma_{J}(M)\right)$. Hence, it is clear that

$$
\text { Ann } M / \Gamma_{J}(M) \subseteq \operatorname{Ann} H_{I}^{1}\left(M / \Gamma_{J}(M)\right)=\operatorname{Ann} H_{I}^{1}(M) .
$$

Moreover, since we have $H_{I}^{1}\left(M / \Gamma_{J}(M)\right) \cong H_{I}^{1}(M) \neq 0$ and $\operatorname{Supp} M / \Gamma_{J}(M) \subseteq$ Supp $M$, it follows from [5, Theorem 2.2] that $\operatorname{cd}\left(I, M / \Gamma_{J}(M)\right)=\operatorname{cd}(I, M)=1$. Now, it is enough to prove

$$
\operatorname{Ann} H_{I}^{1}\left(M / \Gamma_{J}(M)\right) \subseteq \operatorname{Ann} M / \Gamma_{J}(M) .
$$

To do this, assume the contrary. Then there is an element

$$
x \in\left(\operatorname{Ann} H_{I}^{1}\left(M / \Gamma_{J}(M)\right) \backslash \operatorname{Ann} M / \Gamma_{J}(M)\right) .
$$

Let $N:=M / \Gamma_{J}(M)$. Then, since $\operatorname{Ass}_{R} N=\left(\operatorname{Ass}_{R} M \backslash V(J)\right) \subseteq\left(\operatorname{Ass}_{R} M \backslash V(I)\right)$ it follows that $\operatorname{Ass}_{R} \Gamma_{I}(N)=\operatorname{Ass}_{R} N \cap V(I)=\emptyset$ and hence $\Gamma_{I}(N)=0$. Next, consider the following two exact sequences:

$$
0 \rightarrow 0: N \quad x \stackrel{f}{\rightarrow} x N \rightarrow 0 \text { and } 0 \rightarrow x N \stackrel{g}{\rightarrow} N \rightarrow N / x N \rightarrow 0,
$$

where $g \circ f$ is the map $N \stackrel{x}{\rightarrow} N$. Since $x \in \operatorname{Ann} H_{I}^{1}(N)$, by the definition we have $x H_{I}^{1}(N)=0$. In particular, the map

$$
H_{I}^{1}(g) \circ H_{I}^{1}(f)=H_{I}^{1}(g \circ f): H_{I}^{1}(N) \stackrel{x}{\rightarrow} H_{I}^{1}(N)
$$

is the zero homomorphism. Moreover, the exact sequences (2.9.1) yield the exact sequences:

$$
H_{I}^{1}(N) \stackrel{H_{I}^{1}(f)}{\longrightarrow} H_{I}^{1}(x N) \longrightarrow H_{I}^{2}\left(0:_{N} x\right)
$$

and

$$
H_{I}^{0}(N / x N) \longrightarrow H_{I}^{1}(x N) \stackrel{H_{I}^{1}(g)}{\longrightarrow} H_{I}^{1}(N) .
$$

Since Supp $0::_{N} x \subseteq \operatorname{Supp} N \subseteq \operatorname{Supp} M$, it follows from [5, Theorem 2.2] that

$$
\operatorname{cd}\left(I, 0:_{N} x\right) \leq \operatorname{cd}(I, M)=1 \text {. }
$$

So, by the definition we have $H_{I}^{2}\left(0:_{N} x\right)=0$. Therefore, from the exact sequence (2.9.2), it follows that the map $H_{I}^{1}(f)$ is an epimorphism and hence from the fact that $H_{I}^{1}(g) \circ H_{I}^{1}(f)=0$ it follows that $H_{I}^{1}(g)$ is the zero map. So, from the exact sequence (2.9.3) we get the following exact sequence

$$
H_{I}^{0}(N / x N) \rightarrow H_{I}^{1}(x N) \rightarrow 0,
$$

which implies the $R$-module $H_{I}^{1}(x N)$ is finitely generated. Then, we claim that $H_{I}^{1}(x N)=0$. Assume the contrary. Then, since $H_{I}^{1}(x N) \neq 0$ it follows that Supp $H_{I}^{1}(x N) \neq \emptyset$. Let $\mathfrak{q} \in \operatorname{Supp} H_{I}^{1}(x N)$. Then, using [5, Theorem 2.2] and 
[4, Theorem 4.3.2], it is straightforward to see that $\operatorname{cd}\left(I R_{\mathfrak{q}},(x N)_{\mathfrak{q}}\right)=1$. So, by Lemma 2.8 the $R_{\mathfrak{q}}$-module

$$
H_{I R_{\mathfrak{q}}}^{1}\left((x N)_{\mathfrak{q}}\right) \cong\left(H_{I}^{1}(x N)\right)_{\mathfrak{q}}
$$

is not finitely generated, which is a contradiction. Thus, we have $H_{I}^{1}(x N)=0$. Moreover, as $\operatorname{Supp} x N \subseteq \operatorname{Supp} M,\left[5\right.$, Theorem 2.2] implies that $H_{I}^{i}(x N)=0$ for all integers $i \geq 2$. Also, we have $\Gamma_{I}(x N) \subseteq \Gamma_{I}(N)=0$. So we have $H_{I}^{i}(x N)=0$ for all integers $i$. Now, it follows from [4, Therem 2.6.7] that, $I x N=x N$ and hence we have $I+\operatorname{Ann} x N=R$. Since by the hypothesis, we have $x \notin 0::_{R} N$, it is clear that $x N \neq 0$. So, there exists an element $\mathfrak{p} \in$ $\operatorname{Ass}_{R} x N$. Now, we have $R=I+\operatorname{Ann} x N \subseteq I+\mathfrak{p} \subseteq R$, and so, $I+\mathfrak{p}=R$. But $\mathfrak{p} \in \operatorname{Ass}_{R} x N \subseteq \operatorname{Ass}_{R} N=\operatorname{Ass}_{R} M \backslash V(J)$. Since, $\mathfrak{p} \in \operatorname{Ass}_{R} M$ and $I+\mathfrak{p}=R$ it follows that $\mathfrak{p} \in B \subseteq V(J)$. On the other hand, we have $\mathfrak{p} \in\left(\operatorname{Ass}_{R} M \backslash V(J)\right)$, which means $\mathfrak{p} \notin V(J)$. This is a contradiction.

The following result follows from Theorem 2.9.

Corollary 2.10. Let $(R, \mathfrak{m})$ be a Noetherian local ring, $I$ an ideal of $R$ and $M$ be a finitely generated $R$-module. If $\operatorname{cd}(I, M)=1$, then $\operatorname{Ann} H_{I}^{1}(M)=$ Ann $M / \Gamma_{I}(M)$. In particular, $\operatorname{dim} R / \operatorname{Ann} H_{I}^{1}(M)=\operatorname{dim} M / \Gamma_{I}(M)$.

Proof. Let $A=\operatorname{Ass}_{R} M \cap V(I)$ and $B=\left\{\mathfrak{p} \in \operatorname{Ass}_{R} M: \mathfrak{p}+I=R\right\}$. By Theorem 2.9 we have Ann $H_{I}^{1}(M)=\operatorname{Ann} M / \Gamma_{J}(M)$, where $J=\bigcap_{\mathfrak{p} \in(A \cup B)} \mathfrak{p}$. Since, $R$ is local, it is clear that $B=\emptyset$. So, we have $J=\bigcap_{\mathfrak{p} \in A} \mathfrak{p}$. Now, it is straightforward and left to reader, that in this situation we have $\Gamma_{J}(M)=$ $\Gamma_{I}(M)$.

\section{Exactness of ideal transforms}

In this section we will consider the exactness of the $I$-transform functor $D_{I}(-)$. The main results of this section are Theorems 3.5, 3.6 and 3.12. But, first we need the following Lemmata.

Lemma 3.1. Let $R$ be a Noetherian local ring and $I=\left(x_{1}, \ldots, x_{n}\right)$ be an ideal of $R$ and let $M$ be an $R$-module. Let $\mathscr{S}$ be a Serre subcategory of the category of $R$-modules. Then the following statements are equivalent:

(i) The $R$-module $\operatorname{Ext}_{R}^{i}(R / I, M)$ belongs to $\mathscr{S}$ for all integers $i \geq 0$,

(ii) The $R$-module $\operatorname{Tor}_{i}^{R}(R / I, M)$ belongs to $\mathscr{S}$ for all integers $i \geq 0$,

(iii) The Koszul cohomology module $H^{i}\left(x_{1}, \ldots, x_{n} ; M\right)$ belongs to $\mathscr{S}$ for all integers $i=0, \ldots, n$.

Proof. Follows from the method of the proof [14, Theorem 2.1].

Lemma 3.2. Let $R$ be a Noetherian local ring and $I$ a proper non-zero ideal of $R$. Then the following statements are equivalent:

(i) The I-transform functor $D_{I}(-)$ is exact,

(ii) $D_{I}(R)=I D_{I}(R)$, 
(iii) $\operatorname{cd}(I, R) \leq 1$,

(iv) $H_{I}^{n}\left(D_{I}(R)\right)=0$ for all integers $n \geq 0$,

(v) $\operatorname{Ext}_{R}^{n}\left(R / I, D_{I}(R)\right)=0$ for all integers $n \geq 0$,

(vi) $\operatorname{Tor}_{n}^{R}\left(R / I, D_{I}(R)\right)=0$ for all integers $n \geq 0$.

Proof. (i) $\Leftrightarrow$ (ii) See [4, Proposition 6.3.5].

(i) $\Leftrightarrow$ (iii) See [4, Lemma 6.3.1].

(iii) $\Rightarrow$ (iv) By $\left[4\right.$, Corollary 2.2.8(iv)], we have $H_{I}^{n}\left(D_{I}(R)\right)=0$ for $n=0,1$ and by $[4$, Corollary $2.2 .8(\mathrm{v})]$, we have $H_{I}^{n}\left(D_{I}(R)\right) \cong H_{I}^{n}(R)=0$ for all integers $n \geq 2$.

(iv) $\Rightarrow$ (v) Follows applying [14, Proposition 3.9] to the zero Serre category.

$(\mathrm{v}) \Rightarrow$ (vi) Follows applying Lemma 3.1, getting $\mathscr{S}$ equal to the zero Serre category.

(vi) $\Rightarrow$ (ii) Since $D_{I}(R)=I D_{I}(R)$ if and only if $\operatorname{Tor}_{0}^{R}\left(R / I, D_{I}(R)\right)=0$, the assertion is clear.

Lemma 3.3. Let $R$ be a Noetherian ring (not necessarily local) and $I$ and $J$ be ideals of $R$, and $M$ be a non-zero finitely generated $R$-module, such that $(I+J) M \neq M$. Then

$$
\operatorname{cd}(I+J, M) \leq \operatorname{cd}(I, M)+\operatorname{cd}(J, M) .
$$

Proof. See [6, Proposition 2.13].

Corollary 3.4. Let $R$ be a Noetherian ring (not necessarily local) and $I$ and $J$ be ideals of $R$, and $M$ be a non-zero finitely generated $R$-module, such that $(I+J) M \neq M, \operatorname{cd}(I, M) \leq 1$ and $\operatorname{cd}(J, M) \leq 1$. Then $\operatorname{cd}(I \cap J, M) \leq 1$.

Proof. By Lemma 3.3 we have $\operatorname{cd}(I+J, M) \leq 2$. Now the assertion follows from the Mayer-Vietoris exact sequence, (see [4, Theorem 3.2.3]).

Theorem 3.5. Let $R$ be a Noetherian local ring and $I_{1}, I_{2}, \ldots, I_{n}(n \geq 2)$ be ideals of $R$ such that for each $1 \leq j \leq n$, the $I_{j}$-transform functor $D_{I_{j}}(-)$ is exact. Let $\mathfrak{a}=\cap_{j=1}^{n} I_{j}$. Then, the $\mathfrak{a}$-transform functor $D_{\mathfrak{a}}(-)$ is exact.

Proof. Since, for each $1 \leq j \leq n$, the $I_{j}$-transform functor $D_{I_{j}}(-)$ is exact it follows from Lemma 3.2 that, for each $1 \leq j \leq n$, we have $\operatorname{cd}\left(I_{j}, R\right) \leq 1$. Now, using Corollary 3.4 and by induction on $n$ it is easy to see that $\operatorname{cd}\left(\cap_{j=1}^{n} I_{j}, R\right) \leq$ 1. Now the assertion follows from Lemma 3.2.

Theorem 3.6. Let $R$ be a Noetherian local ring and $I$ be an ideal of $R$ such that for each minimal prime ideal $\mathfrak{p}$ of $I$, the $\mathfrak{p}$-transform functor $D_{\mathfrak{p}}(-)$ is exact. Then the I-transform functor $D_{I}(-)$ is exact.

Proof. Follows from Theorem 3.5.

The following lemmata are needed in the proof of Theorem 2.12. 
Lemma 3.7. Let $R$ be a Noetherian local ring and $I$ be an ideal of $R$ and let $M$ be a finitely generated $R$-module. Then, $\operatorname{Ass}_{R} D_{I}(M)=\operatorname{Ass}_{R} M / \Gamma_{I}(M)$. In particular, the set $\operatorname{Ass}_{R} D_{I}(M)$ is finite and $\operatorname{Ass}_{R} D_{I}(M) \cap V(I)=\emptyset$.

Proof. By [4, Remark 2.2.7], there is an exact sequence

$$
0 \rightarrow M / \Gamma_{I}(M) \rightarrow D_{I}(M) \rightarrow H_{I}^{1}(M) \rightarrow 0,
$$

which implies

$$
\operatorname{Ass}_{R} M / \Gamma_{I}(M) \subseteq \operatorname{Ass}_{R} D_{I}(M) \subseteq\left(\operatorname{Ass}_{R} M / \Gamma_{I}(M) \bigcup \operatorname{Ass}_{R} H_{I}^{1}(M)\right) .
$$

But, since the local cohomology module $H_{I}^{1}(M)$ is $I$-torsion it follows that $\operatorname{Ass}_{R} H_{I}^{1}(M) \subseteq V(I)$. On the other hand, in view of [4, Corollary 2.2.8(iv)], we have $\Gamma_{I}\left(D_{I}(M)\right)=0$, which implies $\operatorname{Ass}_{R} D_{I}(M) \cap V(I)=\operatorname{Ass}_{R} \Gamma_{I}\left(D_{I}(M)\right)=$ $\emptyset$ and hence,

$$
\operatorname{Ass}_{R} D_{I}(M) \cap \operatorname{Ass}_{R} H_{I}^{1}(M)=\emptyset .
$$

Now, it is clear that $\operatorname{Ass}_{R} D_{I}(M)=\operatorname{Ass}_{R} M / \Gamma_{I}(M)$.

Recall that a sequence $x_{1}, \ldots, x_{r}$ of elements in the ideal $I$ of $R$ is said to be an $I$-filter regular sequence for $R$, if

$$
x_{i} \notin \mathfrak{p} \text { for all } \mathfrak{p} \in \operatorname{Ass}_{R} R /\left(x_{1}, \ldots, x_{i-1}\right) / \Gamma_{I}\left(R /\left(x_{1}, \ldots, x_{i-1}\right)\right)
$$

for all $i=1, \ldots, r$. The concept of an $I$-filter regular sequence is an extension of the better known notion of regular sequence and was introduced by Schenzel et al. [20].

Lemma 3.8. Let $I$ be a non-nilpotent proper ideal of the Noetherian ring $R$ with $\operatorname{ara}(I)=n$. Then there exists an $I$-filter regular sequence $y_{1}, \ldots, y_{n}$ for $R$ such that $\operatorname{Rad}(I)=\operatorname{Rad}\left(y_{1}, \ldots, y_{n}\right)$.

Proof. See [13, Proposition 2.1].

Lemma 3.9. Let I be a non-nilpotent proper ideal of the Noetherian local ring $(R, \mathfrak{m})$ with $\operatorname{ara}(I)=n \geq 1$. Then, the $I$-transform functor $D_{I}(-)$ is exact if and only if there is an I-filter regular sequence $y_{1}, \ldots, y_{n}$ for $R$ such that $\operatorname{Rad}(I)=\operatorname{Rad}\left(y_{1}, \ldots, y_{n}\right)$ and $y_{1}, \ldots, y_{n-1}$ is an $D_{I}(R)$-regular sequence and the map

$$
\zeta: D_{I}(R) /\left(y_{1}, \ldots, y_{n-1}\right) D_{I}(R) \stackrel{y_{n}}{\rightarrow} D_{I}(R) /\left(y_{1}, \ldots, y_{n-1}\right) D_{I}(R)
$$

is an isomorphism.

Proof. " $\Leftarrow "$ ". If there is an $I$-filter regular sequence $y_{1}, \ldots, y_{n}$ for $R$ such that $\operatorname{Rad}(I)=\operatorname{Rad}\left(y_{1}, \ldots, y_{n}\right)$ and $y_{1}, \ldots, y_{n-1}$ is an $D_{I}(R)$-regular sequence and the map

$$
\zeta: D_{I}(R) /\left(y_{1}, \ldots, y_{n-1}\right) D_{I}(R) \stackrel{y_{n}}{\rightarrow} D_{I}(R) /\left(y_{1}, \ldots, y_{n-1}\right) D_{I}(R)
$$

is an isomorphism, then it is clear that $\left(y_{1}, \ldots, y_{n}\right) D_{I}(R)=D_{I}(R)$. Now, since $\left(y_{1}, \ldots, y_{n}\right) \subseteq I$, it follows that

$$
D_{I}(R)=\left(y_{1}, \ldots, y_{n}\right) D_{I}(R) \subseteq I D_{I}(R) \subseteq D_{I}(R),
$$


which implies that $I D_{I}(R)=D_{I}(R)$ and so by Lemma 3.2 , the $I$-transform functor $D_{I}(-)$ is exact.

" $\Rightarrow$ ". Let the $I$-transform functor $D_{I}(-)$ is exact. Then, by Lemma 3.2 we have $I D_{I}(R)=D_{I}(R)$. Now, using induction it is easy to see that for every positive integer $k$, we have $I^{k} D_{I}(R)=D_{I}(R)$. On the other hand, by Lemma 3.8, there exists an $I$-filter regular sequence $y_{1}, \ldots, y_{n}$ for $R$ such that $\operatorname{Rad}(I)=\operatorname{Rad}\left(y_{1}, \ldots, y_{n}\right)$. By the definition there exists a positive integer $\ell$ such that $I^{\ell} \subseteq\left(y_{1}, \ldots, y_{n}\right)$ and so

$$
D_{I}(R)=I^{\ell} D_{I}(R) \subseteq\left(y_{1}, \ldots, y_{n}\right) D_{I}(R) \subseteq D_{I}(R),
$$

which implies $\left(y_{1}, \ldots, y_{n}\right) D_{I}(R)=D_{I}(R)$. Moreover, since $\operatorname{ara}(I)=n$ it follows from definition that

$$
\operatorname{Rad}(I)=\operatorname{Rad}\left(y_{1}, \ldots, y_{n}\right) \neq \operatorname{Rad}\left(y_{1}, \ldots, y_{n-1}\right)
$$

and so, the ideal $\left(y_{1}, \ldots, y_{n}\right) /\left(y_{1}, \ldots, y_{n-1}\right)$ of the local ring $R /\left(y_{1}, \ldots, y_{n-1}\right)$ is not nilpotent. This means that

$$
T / \Gamma_{\left(y_{1}, \ldots, y_{n}\right)}(T) \neq 0,
$$

where $T:=R /\left(y_{1}, \ldots, y_{n-1}\right)$. Therefore, since $R$ is local it follows from [4, Theorem 6.2.7] that, $\operatorname{cd}\left(\left(y_{1}, \ldots, y_{n}\right), T / \Gamma_{\left(y_{1}, \ldots, y_{n}\right)}(T)\right) \geq 1$. (Note that $\Gamma_{\left(y_{1}, \ldots, y_{n}\right)}\left(T / \Gamma_{\left(y_{1}, \ldots, y_{n}\right)}(T)\right)=0$.) Hence, using [4, Corollary 2.1.7], it follows that $\operatorname{cd}\left(\left(y_{1}, \ldots, y_{n}\right), T\right) \geq 1$. On the other hand, using [5, Theorem 2.2] and Lemma 3.2, we have

$$
\operatorname{cd}\left(\left(y_{1}, \ldots, y_{n}\right), T\right)=\operatorname{cd}(I, T) \leq \operatorname{cd}(I, R) \leq 1,
$$

which implies that $\operatorname{cd}\left(\left(y_{1}, \ldots, y_{n}\right), T\right)=1$. In particular,

$$
H_{I}^{1}(T)=H_{\left(y_{1}, \ldots, y_{n}\right)}^{1}(T) \neq 0 .
$$

By [4, Remark 2.2.7], there is an exact sequence

$$
0 \rightarrow R / \Gamma_{I}(R) \rightarrow D_{I}(R) \rightarrow H_{I}^{1}(R) \rightarrow 0,
$$

which using [4, Exercise 6.1.8] and effecting the functor $-\otimes_{R} T$, yields the exact sequence

$$
D_{I}(R) /\left(y_{1}, \ldots, y_{n-1}\right) D_{I}(R) \rightarrow H_{I}^{1}(T) \rightarrow 0 .
$$

Now, as $H_{I}^{1}(T) \neq 0$, it follows from the last exact sequence that

$$
D_{I}(R) /\left(y_{1}, \ldots, y_{n-1}\right) D_{I}(R) \neq 0 .
$$

In particular, $\left(y_{1}, \ldots, y_{n-1}\right) D_{I}(R) \neq D_{I}(R)$. Also, in view of $[4$, Exercise 6.1.8], we have

$$
\begin{aligned}
D_{I}(R) /\left(y_{1}, \ldots, y_{j-1}\right) D_{I}(R) & \simeq D_{I}(R) \otimes_{R} R /\left(y_{1}, \ldots, y_{j-1}\right) \\
& \simeq D_{I}\left(R /\left(y_{1}, \ldots, y_{j-1}\right)\right)
\end{aligned}
$$


for each $1 \leq j \leq n$. Next, it follows from the definition of $I$-filter regular sequence $y_{1}, \ldots, y_{n}$ for $R$ and Lemma 3.7, that for each $1 \leq j \leq n$ the map

$$
D_{I}(R) /\left(y_{1}, \ldots, y_{j-1}\right) D_{I}(R) \stackrel{y_{j}}{\rightarrow} D_{I}(R) /\left(y_{1}, \ldots, y_{j-1}\right) D_{I}(R)
$$

is a monomorphism. Now, it is clear that, $y_{1}, \ldots, y_{n-1}$ is an $D_{I}(R)$-regular sequence and the map

$$
\zeta: D_{I}(R) /\left(y_{1}, \ldots, y_{n-1}\right) D_{I}(R) \stackrel{y_{n}}{\rightarrow} D_{I}(R) /\left(y_{1}, \ldots, y_{n-1}\right) D_{I}(R)
$$

is an isomorphism.

Lemma 3.10. Let I be a non-nilpotent proper ideal of the Noetherian local ring $(R, \mathfrak{m})$ such that the I-transform functor $D_{I}(-)$ is exact. Then, $D_{I}(R)$ is a flat $R$-module.

Proof. Since $I$ is a non-nilpotent proper ideal of $R$, it follows that $\operatorname{ara}(I) \geq 1$. Let $\operatorname{ara}(I)=n$. Then, in view of Lemma 3.9, there are elements $y_{1}, \ldots, y_{n} \in I$, such that $\operatorname{Rad}(I)=\operatorname{Rad}\left(y_{1}, \ldots, y_{n}\right), y_{1}, \ldots, y_{n-1}$ is an $D_{I}(R)$-regular sequence and the map

$$
\zeta: D_{I}(R) /\left(y_{1}, \ldots, y_{n-1}\right) D_{I}(R) \stackrel{y_{n}}{\rightarrow} D_{I}(R) /\left(y_{1}, \ldots, y_{n-1}\right) D_{I}(R)
$$

is an isomorphism. We must show that $\operatorname{Tor}_{1}^{R}\left(N, D_{I}(R)\right)=0$ for each $R$ module $N$. Assume the contrary. Then there is an $R$-module $M$ such that $\operatorname{Tor}_{1}^{R}\left(M, D_{I}(R)\right) \neq 0$. The exact sequence

$$
0 \rightarrow \Gamma_{I}(R) \rightarrow R \rightarrow R / \Gamma_{I}(R) \rightarrow 0,
$$

yields the exact sequence

$$
\operatorname{Tor}_{1}^{R}(M, R) \rightarrow \operatorname{Tor}_{1}^{R}\left(M, R / \Gamma_{I}(R)\right) \rightarrow \operatorname{Tor}_{0}^{R}\left(M, \Gamma_{I}(R)\right) .
$$

But, since $R$ is a flat $R$-module, we have $\operatorname{Tor}_{1}^{R}(M, R)=0$. So, the last exact sequence can be viewed as follows:

$$
0 \rightarrow \operatorname{Tor}_{1}^{R}\left(M, R / \Gamma_{I}(R)\right) \rightarrow \operatorname{Tor}_{0}^{R}\left(M, \Gamma_{I}(R)\right) .
$$

In particular, since $\operatorname{Tor}_{0}^{R}\left(M, \Gamma_{I}(R)\right)$ is an $R$-module with

$$
\operatorname{Supp} \operatorname{Tor}_{0}^{R}\left(M, \Gamma_{I}(R)\right) \subseteq \operatorname{Supp} \Gamma_{I}(R) \subseteq V(I),
$$

it follows that the $R$-module $\operatorname{Tor}_{1}^{R}\left(M, R / \Gamma_{I}(R)\right)$ is $I$-torsion. Moreover, for $j \geq 2$, the exact sequence (3.10.1) yields the isomorphism

$$
\operatorname{Tor}_{j}^{R}\left(M, R / \Gamma_{I}(R)\right) \cong \operatorname{Tor}_{j-1}^{R}\left(M, \Gamma_{I}(R)\right),
$$

which by similar argument implies that the $R$-module $\operatorname{Tor}_{j}^{R}\left(M, R / \Gamma_{I}(R)\right)$ is $I$-torsion, for all integers $j \geq 2$. Moreover, by [4, Remark 2.2.7], there is an exact sequence

$$
0 \rightarrow R / \Gamma_{I}(R) \rightarrow D_{I}(R) \rightarrow H_{I}^{1}(R) \rightarrow 0,
$$

which for all for $j \geq 1$, induces an exact sequence

$$
\operatorname{Tor}_{j}^{R}\left(M, R / \Gamma_{I}(R)\right) \rightarrow \operatorname{Tor}_{j}^{R}\left(M, D_{I}(R)\right) \rightarrow \operatorname{Tor}_{j}^{R}\left(M, H_{I}^{1}(R)\right) .
$$


Since, we have $\operatorname{Supp} \operatorname{Tor}_{j}^{R}\left(M, H_{I}^{1}(R)\right) \subseteq \operatorname{Supp} H_{I}^{1}(R) \subseteq V(I)$, it follows that the $R$-module $\operatorname{Tor}_{j}^{R}\left(M, H_{I}^{1}(R)\right)$ is $I$-torsion. Now, since the $R$-modules

$$
\operatorname{Tor}_{j}^{R}\left(M, R / \Gamma_{I}(R)\right) \quad \text { and } \quad \operatorname{Tor}_{j}^{R}\left(M, H_{I}^{1}(R)\right)
$$

are $I$-torsion, the exact sequence (3.10.3) implies that the $R$-module $\operatorname{Tor}_{j}^{R}(M$, $\left.D_{I}(R)\right)$ is $I$-torsion, for all integers $j \geq 1$. Now, let $D:=D_{I}(R)$. The exact sequence

$$
0 \rightarrow D \stackrel{y_{1}}{\rightarrow} D \rightarrow D / y_{1} D \rightarrow 0,
$$

induces an exact sequence as

$$
\begin{gathered}
\operatorname{Tor}_{j+1}^{R}(M, D) \stackrel{y_{1}}{\rightarrow} \operatorname{Tor}_{j+1}^{R}(M, D) \\
\rightarrow \operatorname{Tor}_{j+1}^{R}\left(M, D / y_{1} D\right) \rightarrow \operatorname{Tor}_{j}^{R}(M, D) \stackrel{y_{1}}{\rightarrow} \operatorname{Tor}_{j}^{R}(M, D)
\end{gathered}
$$

for all integers $j \geq 0$. The exact sequence (3.10.4) yields the short exact sequence

$$
\begin{aligned}
0 & \rightarrow \operatorname{Tor}_{j+1}^{R}(M, D) / y_{1} \operatorname{Tor}_{j+1}^{R}(M, D) \\
& \rightarrow \operatorname{Tor}_{j+1}^{R}\left(M, D / y_{1} D\right) \rightarrow 0:_{\operatorname{Tor}_{j}^{R}(M, D)} y_{1} \rightarrow 0
\end{aligned}
$$

for all integers $j \geq 0$. Now, it follows from the exact sequence (3.10.5) that the $R$-module $\operatorname{Tor}_{j}^{R}\left(M, D / y_{1} D\right)$ is $I$-torsion, for all integers $j \geq 2$. Moreover, from the exact sequence

$$
0 \rightarrow \operatorname{Tor}_{2}^{R}(M, D) / y_{1} \operatorname{Tor}_{2}^{R}(M, D) \rightarrow \operatorname{Tor}_{2}^{R}\left(M, D / y_{1} D\right) \rightarrow 0::_{\operatorname{Tor}_{1}^{R}(M, D)} y_{1} \rightarrow 0,
$$

it follows that $\operatorname{Tor}_{2}^{R}\left(M, D / y_{1} D\right) \neq 0$. Because, by the hypothesis, the $R$-module $\operatorname{Tor}_{1}^{R}(M, D)$ is non-zero and $I$-torsion and we have $y_{1} \in I$, and therefore we have $0: \operatorname{Tor}_{1}^{R}(M, D) y_{1} \neq 0$. Now, proceeding in the same way, by an inductive argument we can see the $R$-module $\operatorname{Tor}_{n}^{R}\left(M, D /\left(y_{1}, \ldots, y_{n-1}\right) D\right) \neq 0$ and for all integers $j \geq n$, the $R$-module $\operatorname{Tor}_{j}^{R}\left(M, D /\left(y_{1}, \ldots, y_{n-1}\right) D\right)$ is $I$-torsion. Now, let $W:=D /\left(y_{1}, \ldots, y_{n-1}\right) D$. Then, the isomorphism $\zeta: W \stackrel{y_{n}}{\rightarrow} W$, induces an isomorphism

$$
\operatorname{Tor}_{n}^{R}(M, \zeta): \operatorname{Tor}_{n}^{R}(M, W) \stackrel{y_{n}}{\rightarrow} \operatorname{Tor}_{n}^{R}(M, W) .
$$

But, we have $\operatorname{Tor}_{n}^{R}(M, W) \neq 0$ and $\operatorname{Tor}_{n}^{R}(M, W)$ is $I$-torsion and hence, as $y_{n} \in$ $I$, the $R$-module $\operatorname{Tor}_{n}^{R}(M, W)$ is $R y_{n}$-torsion. So, we have $\operatorname{ker}\left(\operatorname{Tor}_{n}^{R}(M, \zeta)\right)=$ $0:_{\operatorname{Tor}_{n}^{R}(M, W)} y_{n} \neq 0$, which is a contradiction.

Theorem 3.11. Let I be a non-nilpotent proper ideal of the Noetherian ring $R$ such that the I-transform functor $D_{I}(-)$ is exact. Then, $D_{I}(R)$ is a flat $R$-module.

Proof. We must show that $\operatorname{Tor}_{1}^{R}\left(N, D_{I}(R)\right)=0$ for each $R$-module $N$. Assume the contrary. Then there is an $R$-module $M$ such that $\operatorname{Tor}_{1}^{R}\left(M, D_{I}(R)\right) \neq 0$. 


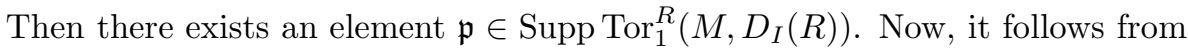
[4, Exercise 4.3.5(iii)] that

$$
\operatorname{Tor}_{1}^{R_{\mathfrak{p}}}\left(M_{\mathfrak{p}}, D_{I R_{\mathfrak{p}}}\left(R_{\mathfrak{p}}\right)\right) \cong \operatorname{Tor}_{1}^{R_{\mathfrak{p}}}\left(M_{\mathfrak{p}},\left(D_{I}(R)\right)_{\mathfrak{p}}\right) \cong\left(\operatorname{Tor}_{1}^{R}\left(M, D_{I}(R)\right)\right)_{\mathfrak{p}} \neq 0
$$

But, in this situation using Lemma 3.2 we have $\operatorname{cd}\left(I R_{\mathfrak{p}}, R_{\mathfrak{p}}\right) \leq \operatorname{cd}(I, R) \leq 1$ and so by Lemma 3.2 the $I R_{\mathfrak{p}}$-transform functor $D_{I R_{\mathfrak{p}}}(-)$ is exact. Also, as $D_{I R_{\mathfrak{p}}}\left(R_{\mathfrak{p}}\right) \neq 0$, it is easy to see that $I R_{\mathfrak{p}}$ is a non-nilpotent proper ideal of the Noetherian local ring $\left(R_{\mathfrak{p}}, \mathfrak{p} R_{\mathfrak{p}}\right)$ and so by Lemma 3.10 the $R_{\mathfrak{p}}$-module $D_{I R_{\mathfrak{p}}}\left(R_{\mathfrak{p}}\right)$ is flat. Therefore, we must have $\operatorname{Tor}_{1}^{R_{\mathfrak{p}}}\left(M_{\mathfrak{p}}, D_{I R_{\mathfrak{p}}}\left(R_{\mathfrak{p}}\right)\right)=0$, which is a contradiction.

Theorem 3.12. Let I be a non-nilpotent proper ideal of the Noetherian ring $R$ such that the I-transform functor $D_{I}(-)$ is exact. Then the ring $D_{I}(R)$ is a finitely generated flat $R$-algebra.

Proof. Follows from [4, Propositions 6.3.4 and 6.3.5] and Theorem 3.11.

Acknowledgements. The author is deeply grateful to the referee for his/her careful reading of the paper and valuable suggestions. Also, the author would like to thank Professor R. Naghipour for his careful reading of the first draft and many helpful suggestions. Finally, the author would like to thank from School of Mathematics, Institute for Research in Fundamental Sciences (IPM) for its financial support.

\section{References}

[1] K. Bahmanpour, Annihilators of local cohomology modules, Comm. Algebra 43 (2015), no. 6, 2509-2515.

[2] K. Bahmanpour, J. A'zami, and G. Ghasemi, On the annihilators of local cohomology modules, J. Algebra 363 (2012), no. 1, 8-13.

[3] K. Bahmanpour and R. Naghipour, Associated primes of local cohomology modules and Matlis duality, J. Algebra 320 (2008), no. 6, 2632-2641.

[4] M. P. Brodmann and R. Y. Sharp, Local Cohomology: an algebraic introduction with geometric applications, Cambridge University Press, Cambridge, 1998.

[5] K. Divaani-Aazar, R. Naghipour, and M. Tousi, Cohomological dimension of certain algebraic varieties, Proc. Amer. Math. Soc. 130 (2002), no. 12, 3537-3544.

[6] G. Ghasemi, K. Bahmanpour, and J. A'zami, Upper bounds for the cohomological dimensions of finitely generated modules over a commutative Noetherian ring, Colloq. Math. 137 (2014), no. 2, 263-270.

[7] A. Grothendieck, Local cohomology, Notes by R. Hartshorne, Lecture Notes in Math., 862, Springer, New York, 1966.

[8] M. Hellus, On the set of Associated primes of a local cohomology module, J. Algebra 237 (2001), no. 1, 406-419.

[9] C. Huneke and J. Koh, Cofiniteness and vanishing of local cohomology modules, Math. Proc. Camb. Phil. Soc. 110 (1991), no. 3, 421-429.

[10] L. R. Lynch, Annihilators of top local cohomology, Comm. Algebra 40 (2012), no. 2, $542-551$.

[11] G. Lyubeznik, Finiteness properties of local cohomology modules (an application of Dmodules to commutative algebra), Invent. Math. 113 (1993), no. 1, 41-55. 
[12] H. Matsumura, Commutative Ring Theory, Cambridge Univ. Press, Cambridge, UK, 1986.

[13] A. A. Mehrvarz, K. Bahmanpour, and R. Naghipour, Arithmetic rank, cohomological dimension and filter regular sequences, J. Algebra Appl. 8 (2009), no. 6, 855-862.

[14] L. Melkersson, Modules cofinite with respect to an ideal, J. Algebra 285 (2005), no. 2, 649-668.

[15] L. T. Nhan and T. N. An, On the unmixedness and universal catenaricity of local rings and local cohomology, J. Algebra 321 (2009), no. 1, 303-311.

[16] L. T. Nhan and T. D. M. Chau, On the top local cohomology modules, J. Algebra 349 (2012), 342-352.

[17] B. Sadeghi, K. Bahmanpour, and J. A'zami, Artinian cofinite modules over complete Noetherian local rings, Czechoslovak Math. J. 63(138) (2013), no. 4, 877-885.

[18] P. Schenzel, Cohomological annihilators, Math. Proc. Camb. Phil. Soc. 91 (1982), no. $3,345-350$.

[19] _ Proregular sequences, local cohomology, and completion, Math. Scand. 92 (2003), no. 2, 161-180

[20] P. Schenzel, N. V. Trung, and N. T. Cuong, Verallgemeinerte Cohen-Macaulay-Moduln, Math. Nachr. 85 (1978), 57-73.

Faculty of Mathematical Sciences

Department of Mathematics

University of Mohaghegh Ardabili

56199-11367, ARDABIL, IRAN

AND

School of Mathematics

Institute For Research in Fundamental Sciences (IPM)

P.O. Box. 19395-5746, Tehran, Iran

E-mail address: bahmanpour.k@gmail.com 